\title{
Symmetry in Electromagnetism
}

\author{
Albert Ferrando ${ }^{1} \mathbb{D}$ and Miguel Ángel García-March ${ }^{2, *}$ \\ 1 Departament d'Òptica, Interdisciplinary Modeling Group, InterTech, Universitat de València, \\ 46100 Burjassot (València), Spain; albert.ferrando@uv.es \\ 2 Instituto Universitario de Matemática Pura y Aplicada, Universitat Politècnica de València, \\ E-46022 València, Spain \\ * Correspondence: garciamarch@mat.upv.es
}

Received: 21 April 2020; Accepted: 22 April 2020; Published: 26 April 2020

check for updates

Electromagnetism plays an essential role, both in basic and applied physics research. The discovery of electromagnetism as the unifying theory for electricity and magnetism represented a cornerstone in modern physics. From the very beginning, symmetry was crucial to the concept of unification: Electromagnetism was soon formulated as a gauge theory, in which a local phase symmetry explained its mathematical formulation. This early connection between symmetry and electromagnetism shows that a symmetry-based approach to many electromagnetic phenomena is recurrent, even today.

Moreover, many crucial technological advances associated with electromagnetism have shaped modern civilization. The control of electromagnetic radiation in nearly all its spectra and scales is still a matter of deep interest. With the advances in material science, even at the nanoscale, the manipulation of matter-radiation interactions has reached unprecedented levels of sophistication. New generations of composite materials present effective electromagnetic properties that permit the molding of electromagnetic radiation in ways that were unconceivable just a few years ago. This is a fertile field for applications and for basic understanding in which symmetry, as in the past, bridges apparently unrelated phenomena, from condensed matter to high-energy physics.

Symmetry is the key tool in the contributions included in this Special Issue. In the context of electromagnetism, the approaches based on symmetry very often lead to diverse treatments of orbital angular momentum or pseudomomentum (as defined in e.g., [1,2]). In this direction, the most sophisticated modern approaches discuss the vectorial case, and in [3], the authors include spin-orbit coupling in nonparaxial fields, and perform a complete an analytical study of the case. The study of electromagnetic knots is also connected to orbital angular momentum, which are a consequence of applying topology concepts to Maxwell equations; in [4] the authors apply symmetry transformations to a particular electromagnetic knot, the hopfion field, to obtain a new set of knotted solutions with the properties of null. Very related to the properties of orbital angular momentum (see [1]) are periodic structures, which play a prominent role in many electromagnetic systems, e.g., microwave and antenna devices. In [5] a method to obtain the relevant transmission, reflection or absorption characteristics of a device obtained from the dispersion diagram are introduced, using general purpose electromagnetic simulation software. Digging deeply into the theory, in [6] the authors present a thorough study of quantum anomalies, which occur when a symmetry of a classical field theory is not also a symmetry of its quantum version. This is discussed in the context of a new example for quantum electromagnetic fields propagating in the presence of gravity, and applications for information extraction ARE foreseen. In this direction, constraint equations in Maxwell theory are discussed in [7]. Interestingly, this work is set in the context of an analogy with constraints of general relativity. A very deep analysis of a fully relativistically covariant and gauge-invariant formulation of classical Maxwell electrodynamics is included in [8], where the authors show the relationship of the symmetry of the inhomogeneous equations obtained and that of Minkowski spacetime. Of a great theoretical interest is also the work presented in [9], where the authors elaborate and improve the previous proposal of a nonlocal action 
functional for electrodynamics depending on the electric and magnetic fields, instead of potentials. They then use this formalism to confront the electric-magnetic duality symmetry of the electromagnetic field and the Aharonov-Bohm effect, two subtle aspects of electrodynamics.

Also, this book includes many applications, such as in sustainable smart buildings [10], or in magnetocardiography, where in [11] the authors present an improved variational mode decomposition model used to decompose the nonstationary signal. The magnetic properties of barium hexaferrite doped with titanium were studied in [12], where the authors propose that they could be used in the recording equipment and permanent magnets. The application to high speed systems is very appealing, such as those related to the Hyperloop concept; in particular in [13], the design and analysis of a plate-type electrodynamic suspension structure for the ground high-speed system is introduced. Finally, a report on the results of research into a vibration-powered milli-or micro-generator is given in [14], where the generators harvest mechanical energy at an optimum level, utilizing the vibration of its mechanical system; here, the authors compare some of the published microgenerator concepts and design versions by using effective power density, among other parameters, and they also provide complementary comments on the applied harvesting techniques.

This book includes papers focusing on detailed and deep theoretical studies to cutting edge applications, with many of the papers includED ALREADY harvesting many citations. The fruitful study of symmetry in electromagnetism continues to offer many encouraging surpriseS, both at a basic and an applied level.

Author Contributions: Both authors contributed equally to this work. All authors have read and agreed to the published version of the manuscript.

Funding: MAGM acknowledges funding from the Spanish Ministry of Education and Vocational Training (MEFP) through the Beatriz Galindo program 2018 (BEAGAL18/00203). A.F. acknowledges funding by the Spanish MINECO grant number TEC2017-86102-C2-1) and Generalitat Valenciana (Prometeo/2018/098).

Conflicts of Interest: The authors declare no conflict of interest.

\section{References}

1. Ferrando. Discrete-symmetry vortices as angular Bloch modes. Phys. Rev. E 2005, 72, 036612. [CrossRef] [PubMed]

2. García-March, M.A.; Ferrando, A.; Zacarés, M.; Vijande, J.; Carr, L.D. Angular pseudomomentum theory for the generalized nonlinear Schrödinger equation in discrete rotational symmetry media. Phys. D Nonlinear Phenom. 2009, 238, 1432-1438. [CrossRef]

3. Arrayás, M.; Trueba, J.L. Spin-Orbital Momentum Decomposition and Helicity Exchange in a Set of Non-Null Knotted Electromagnetic Fields. Symmetry 2018, 10, 88. [CrossRef]

4. Arrayás, M.; Rañada, A.F.; Tiemblo, A.; Trueba, J.L. Null Electromagnetic Fields from Dilatation and Rotation Transformations of the Hopfion. Symmetry 2019, 11, 1105. [CrossRef]

5. Mesa, F.; Rodríguez-Berral, R.; Medina, F. On the Computation of the Dispersion Diagram of Symmetric One-Dimensionally Periodic Structures. Symmetry 2018, 10, 307. [CrossRef]

6. Agulló, I.; del Río, A.; Navarro-Salas, J. On the Electric-Magnetic Duality Symmetry: Quantum Anomaly, Optical Helicity, and Particle Creation. Symmetry 2018, 10, 763. [CrossRef]

7. Rácz, I. On the Evolutionary Form of the Constraints in Electrodynamics. Symmetry 2019, 11, 10. [CrossRef]

8. Majumdar, P.; Ray, A. Maxwell Electrodynamics in Terms of Physical Potentials. Symmetry 2019, 11, 915. [CrossRef]

9. Bernabeu, J.; Navarro-Salas, J. A Non-Local Action for Electrodynamics: Duality Symmetry and the Aharonov-Bohm Effect, Revisited. Symmetry 2019, 11, 1191. [CrossRef]

10. Bravo, J.C.; Castilla, M.V. Geometric Objects: A Quality Index to Electromagnetic Energy Transfer Performance in Sustainable Smart Buildings. Symmetry 2018, 10, 676. [CrossRef]

11. Liao, Y.; He, C.; Guo, Q. Denoising of Magnetocardiography Based on Improved Variational Mode Decomposition and Interval Thresholding Method. Symmetry 2018, 10, 269. [CrossRef]

12. Al Dairy, A.R.; Al-Hmoud, L.A.; Khatatbeh, H.A. Magnetic and Structural Properties of Barium Hexaferrite Nanoparticles Doped with Titanium. Symmetry 2019, 11, 732. [CrossRef] 
13. Guo, Z.; Zhou, D.; Chen, Q.; Yu, P.; Li, J. Design and Analysis of a Plate Type Electrodynamic Suspension Structure for Ground High Speed Systems. Symmetry 2019, 11, 1117. [CrossRef]

14. Szabó, Z.; Fiala, P.; Zukal, J.; Dědková, J.; Dohnal, P. Optimal Structural Design of a Magnetic Circuit for Vibration Harvesters Applicable in MEMS. Symmetry 2020, 12, 110. [CrossRef]

(C) 2020 by the authors. Licensee MDPI, Basel, Switzerland. This article is an open access article distributed under the terms and conditions of the Creative Commons Attribution (CC BY) license (http://creativecommons.org/licenses/by/4.0/). 